\title{
On A One Fixed Point Improved Secant Method for Solving Roots of Polynomials
}

\author{
Bazuaye Frank Etin-Osa and Charles Timinibife Noah \\ Department of Mathematics and Statistics, University of Port Harcourt, \\ Port Harcourt, Rivers State, Nigeria \\ e-mail: etin-osa.bazuaye@ uniport.edu.ng
}

\begin{abstract}
This paper focuses on the construction and implementation of an improved secant method for finding the root of a polynomial. The arithmetic mean in the Marouane's method was replaced by the geometric mean. The result shows that the method converges compete favorably with other methods in literature and efficient as the two points in the conventional secant methods has been reduced to only one fixed point.
\end{abstract}

\section{Introduction}

Over the years, different methods and improvement have been made to the solution of the polynomial equation

$$
P(x)=P_{0}+P_{1} x+P_{2} x^{2}+\cdots+P_{n-1} x^{n-1}+P_{n} x^{n}=0 .
$$

The root-finding problem arises in a wide variety of practical applications in physics and engineering and many others. This problem also has a direct application in the multiple shooting method for two-point boundary-value problems. The basic concept to all root finding numerical methods is iteration or successive approximation. The main idea of an iterative method is to first choose a suitable guess of the root, and then repeatedly improve upon this guess, using some well-defined operations, until we obtain an Received: September 21, 2019; Accepted: December 12, 2019

2010 Mathematics Subject Classification: 65E05, 76Dxx.

Keywords and phrases: root finding, arithmetic mean, geometric mean.

Copyright () 2020 Bazuaye Frank Etin-Osa and Charles Timinibife Noah. This is an open access article distributed under the Creative Commons Attribution License, which permits unrestricted use, distribution, and reproduction in any medium, provided the original work is properly cited. 
approximate root that is sufficiently close to actual root. One of the most widely used root-finding iterative algorithms is Newton's Raphson method.

Brent [1] presented a root finding algorithm by combining the bisection method, the secant method and inverse quadratic interpolation. Hildebrand [2] states that the theory of numerical analysis deals with the location of the roots of an equation. Ostrowski [3] presented the secant method as a modified form of the Newton Ralphson's method. He referred to the secant method as an alteration using successive adjacent points. $\mathrm{He}$ explains that the secant method uses the last two points instead of constantly using one of the initial points. Traub [4] explained that the secant method always uses the last two approximations. He was the first to state that convergence of the secant method is superlinear.

Zheng et al. [5] presented an improvement to Brent's method for finding roots of a function of a single variable. Zhang improvement to Brent's method makes the algorithm earlier presented by Brent to be much simpler and much understandable.

Marouane [6] presented an improvement to the secant method that is much faster in quadratic order with only one initial guess. On his part Chen [7] derived a family of improved secant-like method is proposed. In the paper, the analysis of the convergence shows that this method has super-linear convergence. Efficiency were demonstrated by numerical experiments when the choice of $\alpha$ is correct. Recently also, Tiruneh et al. [8] presented a modification of secant method for finding roots of equations that uses three points for iteration instead of just two. The development of the mathematical formula to be used in the iteration process is provided together with the proof of the rate of convergence which is 1.84 and is the same as the rate of convergence of Mueller's method of root finding. Application examples were given where it was demonstrated that for equations involving ill-conditioned cases, the proposed method has better convergence characteristics compared to the Newton and conventional secant methods.

\section{Materials and Methods}

The secant method is a root-finding algorithm that uses a succession of roots of secant lines to better approximate a root of a function $f(x)=0$. It is an improvement of Newton Ralphson's method where $f^{\prime}\left(x_{n}\right)$ in the Newton-Raphson method is replaced by the difference equation 


$$
f^{\prime}\left(x_{n}\right)=\frac{f\left(x_{n+1}\right)-f\left(x_{n}\right)}{x_{n+1}-x_{n}}
$$

which is the slope of the line through two parts of the curve $y=f(x)$. The conventional secant method is therefore given by the formula:

$$
x_{n+1}=x_{n}-f\left(x_{n}\right) \frac{\left(x_{n}-x_{n-1}\right)}{f\left(x_{n}\right)-f\left(x_{n-1}\right)} .
$$

Marouane [6] improved on (3) by deriving a new method given as

$$
x_{n+1}=x_{n}+\frac{\left|f\left(x_{n}\right)\right|}{2}-\frac{\left|f\left(x_{n}\right)\right|}{2}\left(\frac{f\left(x_{n}+\frac{\left|f\left(x_{n}\right)\right|}{2}\right)}{f\left(x_{n}+\frac{\left|f\left(x_{n}\right)\right|}{2}\right)-f\left(x_{n}\right)}\right) .
$$

\section{Derivation of the new improved methods}

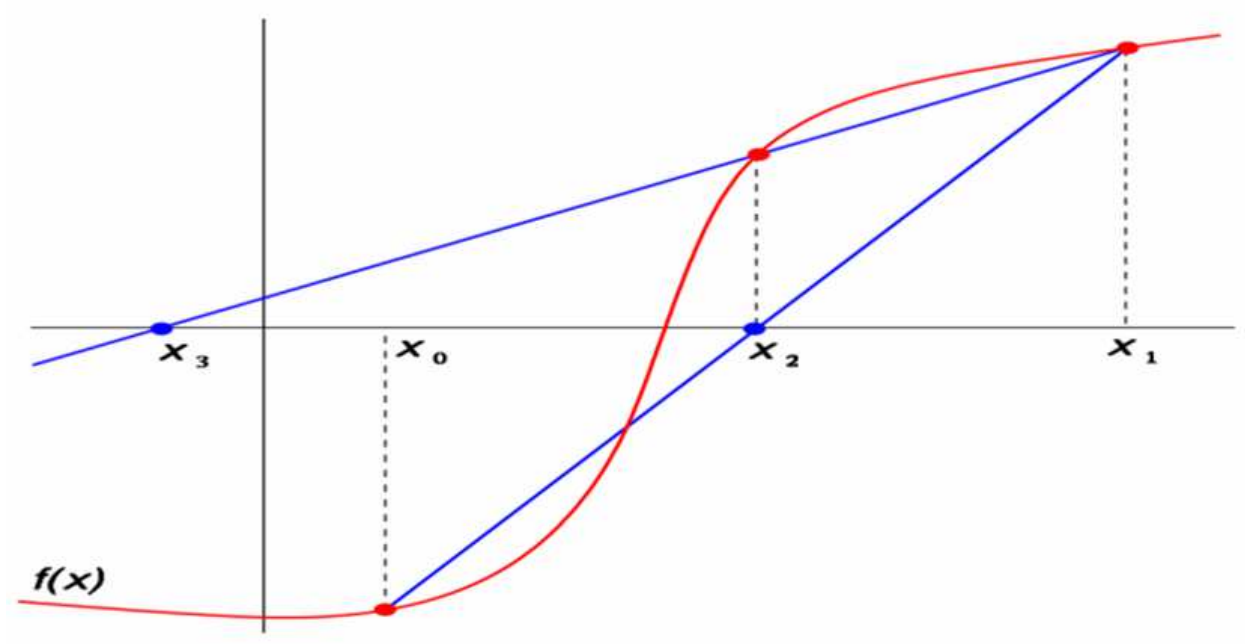

Figure 1. The first two iterations of the secant method. The red curve shows the function $f$, and the blue lines are the secants.

Starting with initial values $x_{0}$ and $x_{1}$, we construct a line through the points $\left(x_{0}, f\left(x_{0}\right)\right)$ and $\left(x_{1}, f\left(x_{1}\right)\right)$, as shown in the picture above. In the gradient intercept form, the equation of this line is $y=m x+c$; that is: 


$$
y=\frac{f\left(x_{1}\right)-f\left(x_{0}\right)}{x_{1}-x_{0}}\left(x-x_{1}\right)+f\left(x_{1}\right) .
$$

The root of this linear function, that is the value of $x$ such that $y=0$ is

$$
x=x_{1}-f\left(x_{1}\right) \frac{x_{1}-x_{0}}{f\left(x_{1}\right)-f\left(x_{0}\right)} .
$$

Maouane replaces

$$
\begin{gathered}
x_{1}=x_{0}+\frac{\left|f\left(x_{0}\right)\right|}{2} \\
f\left(x_{1}\right)=f\left(x_{0}+\frac{\left|f\left(x_{0}\right)\right|}{2}\right)
\end{gathered}
$$

and obtains

$$
x=x_{0}+\frac{\left|f\left(x_{0}\right)\right|}{2}-f\left(x_{0}+\frac{\left|f\left(x_{0}\right)\right|}{2}\right)\left[\frac{x_{0}+\frac{\left|f\left(x_{0}\right)\right|}{2}-x_{0}}{f\left(x_{0}+\frac{\left|f\left(x_{0}\right)\right|}{2}\right)-f\left(x_{0}\right)}\right] .
$$

In this paper, (7) and (8) are replaced as

$$
\begin{gathered}
x_{1} \cong \sqrt{\left|2 x_{0} f\left(x_{0}\right)\right|} \\
f\left(x_{1}\right) \cong f\left(\sqrt{\left|2 x_{0} f\left(x_{0}\right)\right|}\right),
\end{gathered}
$$

respectively.

Substituting equations (10) and (11) into equation (6), we have

$$
x=\sqrt{\left|2 x_{0} f\left(x_{0}\right)\right|}-f\left(\sqrt{\left|2 x_{0} f\left(x_{0}\right)\right|}\right)\left[\frac{\sqrt{\left|2 x_{0} f\left(x_{0}\right)\right|}-x_{0}}{f\left(\sqrt{\left|2 x_{0} f\left(x_{0}\right)\right|}\right)-f\left(x_{0}\right)}\right] .
$$

Generalizing equation (12), we have

$$
x_{n+1}=\sqrt{\left|2 x_{n} f\left(x_{n}\right)\right|}-f\left(\sqrt{\left|2 x_{n} f\left(x_{n}\right)\right|}\right)\left[\frac{\sqrt{\left|2 x_{n} f\left(x_{n}\right)\right|}-x_{n}}{f\left(\sqrt{\left|2 x_{n} f\left(x_{n}\right)\right|}\right)-f\left(x_{n}\right)}\right] .
$$




\section{Numerical Experiments}

In this section, we consider the numerical experiment of the newly improved secant algorithm to ascertain its accuracies and convergence. Also the new algorithm is compared with other existing methods such as Marouane's and conventional secant methods.

Problem 1. Given the equation $f(x)=x^{2}-1$ the roots are given as follows:

Using the new method (13):

When $n=0$, we have

$$
x_{1}=\sqrt{\left|2 x_{0} f\left(x_{0}\right)\right|}-f\left(\sqrt{\left|2 x_{0} f\left(x_{0}\right)\right|}\right)\left[\frac{\sqrt{\left|2 x_{0} f\left(x_{0}\right)\right|}-x_{0}}{f\left(\sqrt{\left|2 x_{0} f\left(x_{0}\right)\right|}\right)-f\left(x_{0}\right)}\right] .
$$

By bisection rule, $f(1)=-1$ and $f(2)=2$. Thus, $f(1) \times f(2)<0$, hence $x \in(1,2)$. It follows that

$$
\begin{aligned}
& x_{0}=\frac{1}{2}(1+2)=1.5 \\
& f(1.5)=0.25, \quad \sqrt{|2(1.5) f(1.5)|}=0.866025404, \quad f(0.8660254040)=-1.25 . \\
& x_{1}=0.866025404+1.25\left(\frac{-0.633974596}{-1.5}\right)=1.1394337567 .
\end{aligned}
$$

When $n=1$,

$$
\begin{aligned}
& x_{2}=\sqrt{\left|2 x_{1} f\left(x_{1}\right)\right|}-f\left(\sqrt{\left|2 x_{1} f\left(x_{1}\right)\right|}\right)\left[\frac{\sqrt{\left|2 x_{1} f\left(x_{1}\right)\right|}-x_{1}}{f\left(\sqrt{\left|2 x_{1} f\left(x_{1}\right)\right|}-f\left(x_{1}\right)\right.}\right], \\
& f\left(x_{1}\right)=-0.055822748, \\
& \sqrt{\left|2 x_{1} f\left(x_{1}\right)\right|}=0.3945522898, \quad f\left(\sqrt{\left|2 x_{1} f\left(x_{1}\right)\right|}\right)=-1.844328491,
\end{aligned}
$$

then

$$
\begin{aligned}
& x_{2}=0.3945522213+1.84432491\left(\frac{-0.9997852773}{-1.788505743}\right), \\
& x_{2}=1.425542813 .
\end{aligned}
$$


When $n=2$,

$$
\begin{aligned}
& x_{3}=\sqrt{\left|2 x_{2} f\left(x_{2}\right)\right|}-f\left(\sqrt{\left|2 x_{2} f\left(x_{2}\right)\right|}\right)\left[\frac{\sqrt{\left|2 x_{2} f\left(x_{2}\right)\right|}-x_{2}}{f\left(\sqrt{\left|2 x_{2} f\left(x_{2}\right)\right|}\right)-f\left(x_{2}\right)}\right], \\
& \sqrt{\left|2 x_{2} f\left(x_{2}\right)\right|}=0.3028630322, \quad f\left(\sqrt{\left|2 x_{2} f\left(x_{2}\right)\right|}\right)=-1.908273984, \\
& f\left(x_{2}\right)=0.032172312,
\end{aligned}
$$

then

$$
\begin{aligned}
& x_{3}=0.302863032+1.908273984\left(\frac{-1.122679781}{-1.940446296}\right)=1.406928949, \\
& x_{3}=0.302863032+1.908273984\left(\frac{-1.122679781}{-1.940446296}\right)=1.406928949 .
\end{aligned}
$$

It continues for the first ten iterations and the results are displayed in the table below:

Table 1. Table of solution generated by the new method.

\begin{tabular}{|c|c|c|c|c|}
\hline $\mathrm{S} / \mathrm{N}$ & $x_{n}$ & $f\left(x_{n}\right)$ & $e_{n}$ & $e_{n+1}$ \\
\hline 1 & 1.394337567 & -0.055822748 & 0.019875995 & 0.011334568 \\
\hline 2 & 1.42554813 & 0.032172312 & 0.011334568 & 0.007284613 \\
\hline 3 & 1.406928949 & -0.0205509344 & 0.007284613 & 0.005190139 \\
\hline 4 & 1.419403701 & 0.014706866 & 0.005190139 & 0.003867308 \\
\hline 5 & 1.410346254 & -0.010923445 & 0.003867308 & 0.003020833 \\
\hline 6 & 1.417234395 & 0.00855333 & 0.003020833 & 0.002416966 \\
\hline 7 & 1.411796596 & -0.006830709 & 0.002416966 & 0.001988036 \\
\hline 8 & 1.416201598 & 0.005626966 & 0.001988036 & 0.001660041 \\
\hline 9 & 1.412553521 & -0.004692552 & 0.001660041 & 0.001411619 \\
\hline 10 & 1.415623181 & 0.003994654 & 0.001411619 & \\
\hline
\end{tabular}


Solving same problem with Marouane is summarized in the table below

Table 2. Table of solution generated by the Marouane's method.

\begin{tabular}{|c|c|c|c|c|}
\hline $\mathrm{S} / \mathrm{N}$ & $x_{n}$ & $f\left(x_{n}\right)$ & $e_{n}$ & $e_{n+1}$ \\
\hline 1 & 1.42000000 & 0.0164 & 0.005786438 & 0.000028415 \\
\hline 2 & 1.41421977 & 0.00008037 & 0.000028415 & 0.000000000 \\
\hline 3 & 1.414213562 & 0.000000001 & 0.000000000 & 0.000000000 \\
\hline 4 & 1.414213562 & 0.000000001 & 0.000000000 & 0.000000000 \\
\hline 5 & 1.414213562 & 0.000000001 & 0.000000000 & 0.000000000 \\
\hline 6 & 1.414213562 & 0.000000001 & 0.000000000 & 0.000000000 \\
\hline 7 & 1.414213562 & 0.000000001 & 0.000000000 & 0.000000000 \\
\hline 8 & 1.414213562 & 0.000000001 & 0.000000000 & 0.000000000 \\
\hline 9 & 1.414213562 & 0.000000001 & 0.000000000 & 0.000000000 \\
\hline 10 & 1.414213562 & 0.000000001 & 0.000000000 & 0.000000000 \\
\hline
\end{tabular}

Also with the conventional secant method as

Table 3. Table of solution generated by the conventional secant method.

\begin{tabular}{|c|c|c|c|c|}
\hline $\mathrm{S} / \mathrm{N}$ & $x_{n}$ & $f\left(x_{n}\right)$ & $e_{n}$ & $e_{n+1}$ \\
\hline 1 & 1.428571429 & 0.040816328 & 0.014357867 & 0.002453105 \\
\hline 2 & 1.416666667 & 0.006944433 & 0.002453105 & 0.000012384 \\
\hline 3 & 1.414225946 & 0.000035028 & 0.000012384 & 0.000000010 \\
\hline 4 & 1.414213572 & 0.000000029 & 0.000000010 & 0.000000000 \\
\hline 5 & 1.414213562 & 0.000000001 & 0.000000000 & 0.000000000 \\
\hline 6 & 1.414213562 & 0.000000001 & 0.000000000 & 0.000000000 \\
\hline 7 & 1.414213562 & 0.000000001 & 0.000000000 & 0.000000000 \\
\hline 8 & 1.414213562 & 0.000000001 & 0.000000000 & 0.000000000 \\
\hline 9 & 1.414213562 & 0.000000001 & 0.000000000 & 0.000000000 \\
\hline 10 & 1.414213562 & 0.000000001 & 0.000000000 & 0.000000000 \\
\hline
\end{tabular}




\section{Results and Discussions}

The summary of the results of solution generated by the different methods is tabulated below:

Table 4. Table of solution generated by the secant method and the new derived method.

\begin{tabular}{|l|l|l|l|l|}
\hline \multicolumn{3}{|c|}{ Conventional Secant Method (SM) } & \multicolumn{2}{c|}{ New Method (NM) } \\
\hline $\mathrm{S} / \mathrm{N}$ & $x_{n}$ & $e_{n}$ & $x_{n}$ & $e_{n}$ \\
\hline 1 & 1.428571429 & 0.014357867 & 1.394337567 & 0.019875995 \\
\hline 2 & 1.416666667 & 0.002453105 & 1.42554813 & 0.011334568 \\
\hline 3 & 1.414225946 & 0.000012384 & 1.406928949 & 0.007284613 \\
\hline 4 & 1.414213572 & 0.000000010 & 1.419403701 & 0.005190139 \\
\hline 5 & 1.414213562 & 0.000000000 & 1.410346254 & 0.003867308 \\
\hline 6 & 1.414213562 & 0.000000000 & 1.417234395 & 0.003020833 \\
\hline 7 & 1.414213562 & 0.000000000 & 1.411796596 & 0.002416966 \\
\hline 8 & 1.414213562 & 0.000000000 & 1.416201598 & 0.001988036 \\
\hline 9 & 1.414213562 & 0.000000000 & 1.412553521 & 0.001660041 \\
\hline 10 & 1.414213562 & 0.000000000 & 1.415623181 & 0.001411619 \\
\hline
\end{tabular}

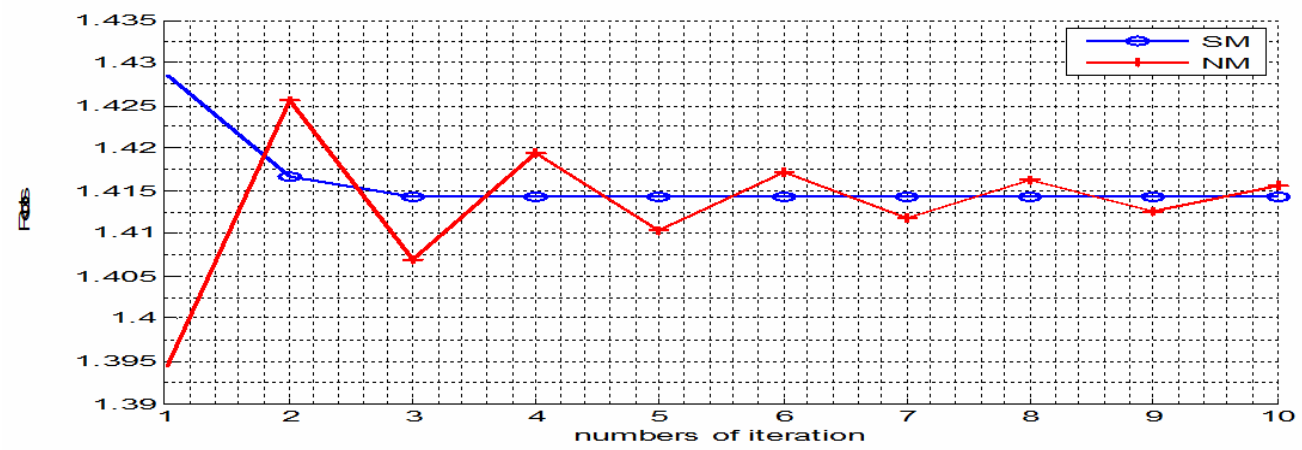

Figure 2. Graph of the conventional secant and improved secant method. 
Table 5. Comparisons between Marouane's method (MA) and the new method (NM).

\begin{tabular}{|l|l|l|l|l|}
\hline \multicolumn{3}{|c|}{ Marouane's Method (MA) } & \multicolumn{2}{c|}{ New Method (NM) } \\
\hline $\mathrm{S} / \mathrm{N}$ & $x_{n}$ & $e_{n}$ & $x_{n}$ & $e_{n}$ \\
\hline 1 & 1.42000000 & 0.005786438 & 1.394337567 & 0.019875995 \\
\hline 2 & 1.41421977 & 0.000028415 & 1.42554813 & 0.011334568 \\
\hline 3 & 1.414213562 & 0.000000000 & 1.406928949 & 0.007284613 \\
\hline 4 & 1.414213562 & 0.000000000 & 1.419403701 & 0.005190139 \\
\hline 5 & 1.414213562 & 0.000000000 & 1.410346254 & 0.003867308 \\
\hline 6 & 1.414213562 & 0.000000000 & 1.417234395 & 0.003020833 \\
\hline 7 & 1.414213562 & 0.000000000 & 1.411796596 & 0.002416966 \\
\hline 8 & 1.414213562 & 0.000000000 & 1.416201598 & 0.001988036 \\
\hline 9 & 1.414213562 & 0.000000000 & 1.412553521 & 0.001660041 \\
\hline 10 & 1.414213562 & 0.000000000 & 1.415623181 & 0.001411619 \\
\hline
\end{tabular}

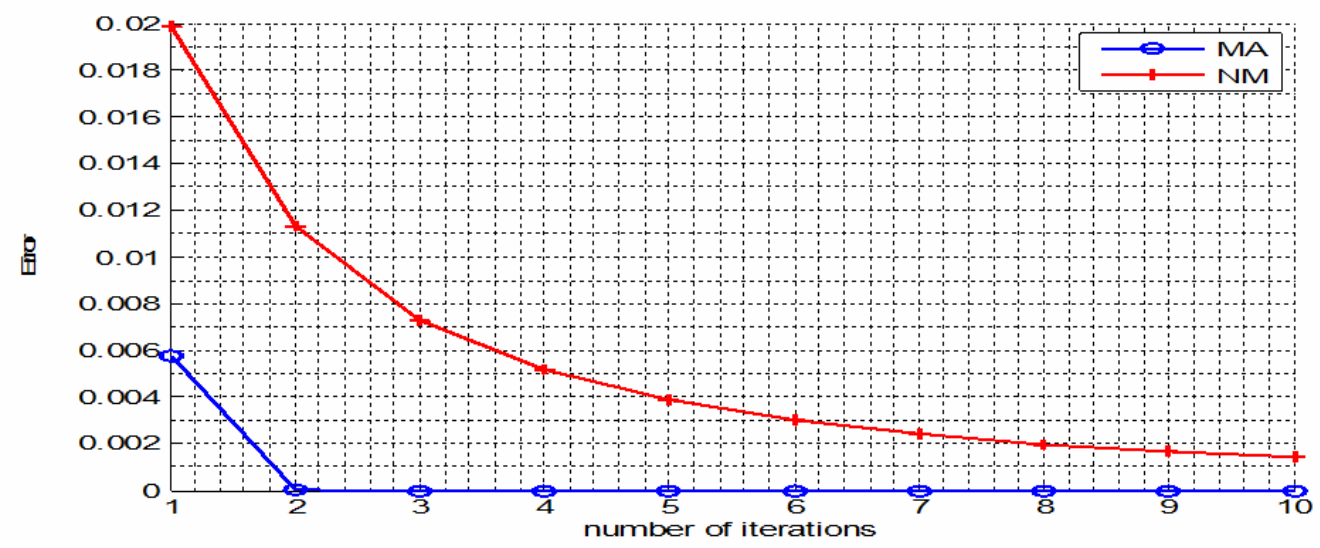

Figure 3. Graph of the Marouane's method (MA) and the new method (NM). 
Table 6. Comparisons between Marouane's method the new method (NM).

\begin{tabular}{|c|c|c|c|c|c|c|}
\hline \multicolumn{2}{|c|}{ Conventional Secant Method (SM) } & \multicolumn{2}{c|}{ Marouane's Method (MA) } & \multicolumn{2}{c|}{ New Method (NM) } \\
\hline $\mathrm{S} / \mathrm{N}$ & $x_{n}$ & $e_{n}$ & $x_{n}$ & $e_{n}$ & $x_{n}$ & $e_{n}$ \\
\hline 1 & 1.428571429 & 0.014357867 & 1.42000000 & 0.005786438 & 1.394337567 & 0.019875995 \\
\hline 2 & 1.416666667 & 0.002453105 & 1.41421977 & 0.000028415 & 1.42554813 & 0.011334568 \\
\hline 3 & 1.414225946 & 0.000012384 & 1.414213562 & 0.000000000 & 1.406928949 & 0.007284613 \\
\hline 4 & 1.414213572 & 0.000000010 & 1.414213562 & 0.000000000 & 1.419403701 & 0.005190139 \\
\hline 5 & 1.414213562 & 0.000000000 & 1.414213562 & 0.000000000 & 1.410346254 & 0.003867308 \\
\hline 6 & 1.414213562 & 0.000000000 & 1.414213562 & 0.000000000 & 1.417234395 & 0.003020833 \\
\hline 7 & 1.414213562 & 0.000000000 & 1.414213562 & 0.000000000 & 1.411796596 & 0.002416966 \\
\hline 8 & 1.414213562 & 0.000000000 & 1.414213562 & 0.000000000 & 1.416201598 & 0.001988036 \\
\hline 9 & 1.414213562 & 0.000000000 & 1.414213562 & 0.000000000 & 1.412553521 & 0.001660041 \\
\hline 10 & 1.414213562 & 0.000000000 & 1.414213562 & 0.000000000 & 1.415623181 & 0.001411619 \\
\hline
\end{tabular}

The graph is presented below

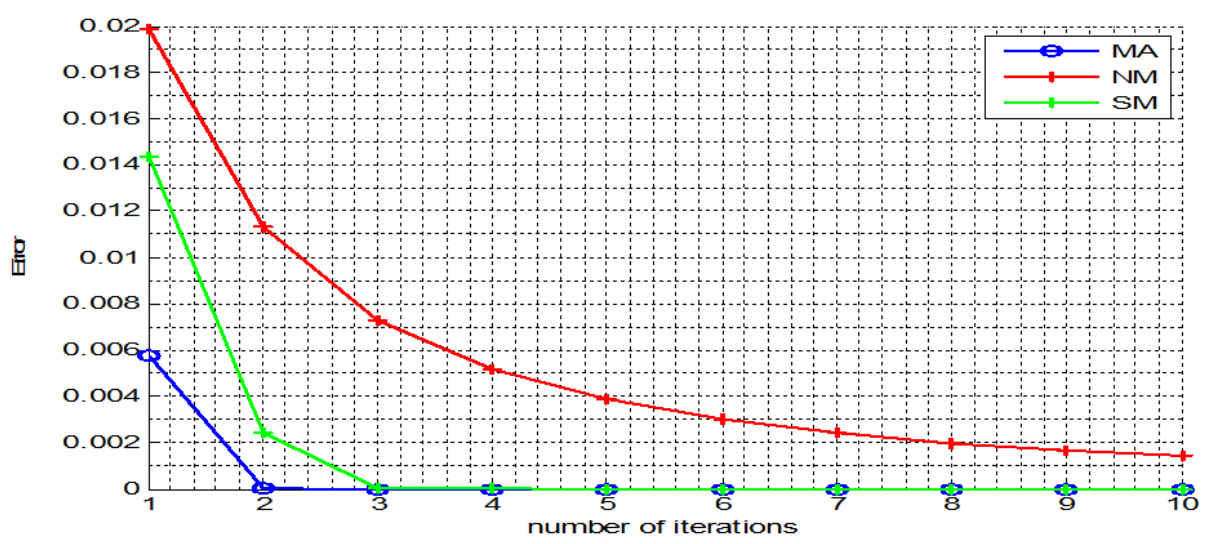

Figure 4. Graph of the Marouane's method, the new method and the conventional secant method.

From the numerical experiment of tables and figures above, it all show that our newly improved secant method converges and it has advantages over the conventional secant method as it requires only one fixed point and hence reduces computational stress. 


\section{Conclusion}

From the analysis, it is discovered that the newly improved secant method converges and requires only one fixed point for computation, which is far better than the conventional secant method that requires two fixed points.

\section{References}

[1] R. P. Brent, Algorithms for Minimization without Derivatives, Prentice-Hall, Englewood Cliffs, NJ, 1973.

[2] F. B. Hildebrand, Introduction to Numerical Analysis, 2nd ed., McGraw-Hill Book Co. Inc., 1974.

[3] A. M. Ostrowski, Solution of Equations and Systems of Equations, Academic Press, NY, 1960.

[4] J. F. Traub, Iterative Methods for the Solution of Equations, Chelsea Publishing Company, New York, 1982.

[5] Q. Zheng, P. Zhao, L. Zhang and W. Ma, Variants of Steffensen-secant method and applications, Appl. Math. Comput. 216(12) (2010), 3486-3496.

https://doi.org/10.1016/j.amc.2010.04.058

[6] R. Marouane, An Improvement to the Secant Method, 2014. http://vixra.org/pdf/1405.0013v1.pdf

[7] Liang Chen, A family of improved secant-like method with super-linear convergence, International Journal of Mathematical and Computational Sciences 7(4) (2013), 635638.

[8] A. T. Tiruneh, T. Y. Debessai, G. C. Bwembya and S. J. Nkambule, A modified threepoint Secant method with improved rate and characteristics of convergence, International Journal of Advances in Mathematics 2019(4) (2019), 69-83. 